\title{
Biochemical Energetics
}

National Cancer Institute

\section{Source}

National Cancer Institute. Biochemical Energetics. NCl Thesaurus. Code C18643.

The study of the energy changes involved in physical and chemical reaction, changes, and systems of living organisms. 